\title{
Objective recording of fetal movements in late pregnancy
}

K. Marß́l, K. Lindström, U. Ulmsten

Fetal movements (FM) are an expression of the physiologic activity of the fetus. During recent years, several reports have shown that a "normal" pattern of FM is related to the fetal well-being. In situations of intrauterine distress, the incidence of FM changes $(1,2)$. However, in most of the reported studies, the results are based on the mothers' subjective evaluation of FM. Such a recording technique may be misleading, since it has been shown that subjective estimation of FM is impaired by considerable errors $(3,4)$, and it has also been discouraged from the use of subjective FM counts in assessing the state of the fetus (5).

Hence a need for an objective method for recording of FM is obvious. Two-dimensional real-time B-mode ultrasound technique is very suitable for observation of fetal motoric activity in early pregnancy. In the second half of pregnancy, however, only a part of the fetal body or fetal extremities can be visualized by this technique. Furthermore, the ultrasound technique is less suitable for long-term recordings and no quantified signals of FM are obtained.

Use of piezo-electric crystals for detection of FM has been previously described(6). We have further developed. this technique and constructed a new device for quantified measurement of FM. Four piezoelectric transducers are placed on the maternal abdominal wall (Fig. 1a). When the fetus moves, the mechanical energy is converted to electric signals within the trans-

A)

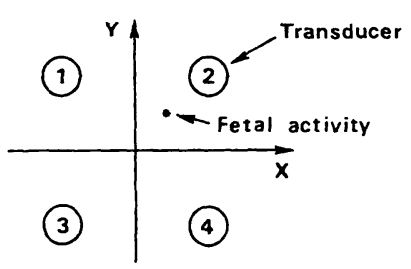

B)

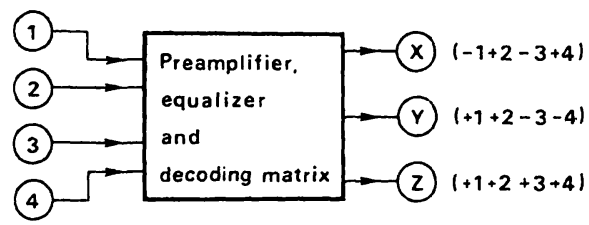

c)

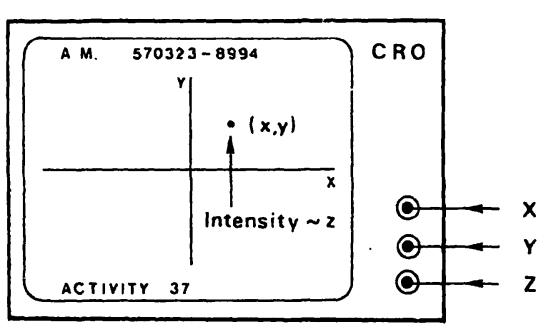
ducers. The signals are transmitted into a special processing unit. By addition and subtraction, outsigmals $X$ and $Y$ are produced (Fig. 1b). The two signals represent the vector, which indicates the centrum of the fetal activity in a co-ordinate system (Fig. 1c). A sum of all four primary signals is equal to the outsignal $\mathrm{Z}$ reflecting the total intensity of the FM.

Fig. 1. Principle of the fetal movement detector. a Localization of the piezo-electric transducers on the maternal abdomen. $\underline{b}$ Processing of the four primary sigmals. c Display of the outsignals $X, Y$, and $Z$ on the oscilloscope screen. The arrow-indicated point represents the centrum of the momentary fetal activity. 
The signals representing the momentary fetal motoric activity are presented on an oscilloscope screen. For each sequence of FM, a new point is displayed. The total activity during a 5-min. period is summed by continuous photographing of the screen. The brightness of the displayed signals is given by the amplitude of the total outsignal $\mathrm{Z}$. The total signal is also recorded graphically and the incidence is measured on-line by an automatic counter.

The new objective method for recording of FM has been compared with the maternal subjective indications of FM and with the simultaneous observations of FM by an ultrasonic real-time scanner. The two objective methods showed an agreement in 92 per cent of registered movements; the mother recognized only 62 per cent of FM recorded by the detector. All the subjectively non-detected FN were those with a relatively low amplitude of the $\mathrm{Z}$ signal. The new method was applied to 25 women with uncomplicated pregnancies between 30 and 34 weeks of gestational age. FM were recorded for $30 \mathrm{~min}$. The mean incidence of FM was 17 per cent of the recording time. A number of FM displayed showed large interindividual variations, ranging 7 to $155 \mathrm{FM}$ episodes within $30 \mathrm{~min}$.

At present, the new FM detector has been introduced in the routine battery of tests used at our Department for supervision of complicated pregnancies. Since the results have not yet been evaluated, no definite conclusions can be drawn concerning the future place of the FM detector in clinics. However, some statements about its usefulness are already now justified: The new detector is non-invasive and offers an objective and quantified measure of FM. It shows a much higher reliability than maternal subjective registration of FM. The method is, in contrast to the ultrasound technique, suitable for long-term recordings and can be applied preferably in late pregnancy. It can be used under clinical conditions and also in physiological studies, e.g. simultaneously with recordings of fetal breathing movements or fetal ECG.

\section{References}

1. PEARSON, J., WEAVER, J.: Fetal activity and fetal well-being: an evaluation. Br. Med. J. 1(1976)1305.

2. SADOVSKY, E., YAFFE, H., POLISHUK, W.Z.: Fetal movement monitoring in normal and pathological pregnancy. Int. J. Gynec. Obstet. 12(1974) 75 .

3. EDWARDS, D., EDWARDS, I.: Fetal movement: development and time course. Science 169(1970)95.

4. GETPINGRR, A.: Comparison between subjective and ultrasound assessments of fetal movement. Br. Med. J. 2(1978)88.

5. WEivDERLEIN, J.M.: Erleben der Fetalbewegungen. Z. Geburtsh. Perinat. $179(1975) 377$.

6. SADOVSKY, E., POLISHUK, W.Z., YAFFE, H., ADLER, D., PACHYS, F., MAHLER, M.: Fetal movements recorder, use and indications. Int. J. Gynec. Obstet. 15(1977) 20. 